\title{
The Development of Supergravity Grand Unification: Circa 1982-85
}

\author{
R. Arnowitt* ${ }^{1}$ A. H. Chamseddine ${ }^{\dagger}, 2,3$ and Pran Nath $^{\ddagger 4}$ \\ ${ }^{1}$ Department of Physics and Astronomy, Texas A\&M University, \\ College Station, Texas 77843-4242, USA \\ ${ }^{2}$ American University of Beirut, Physics Department, Beirut, Lebanon \\ ${ }^{3}$ Institut des Hautes Etudes Scientifique (I.H.E.S.) Bures-sur-Yvette, France \\ ${ }^{4}$ Department of Physics, Northeastern University, Boston, MA 02115, USA
}

(Dated: August 7, 2018)

\begin{abstract}
The development in the early eighties of supergravity grand unified models with gravity mediated breaking of supersymmetry, has led to a remarkable progress in the study of supersymmetry at colliders, in dark matter and in a variety of other experimental searches in the intervening years since that time. The purpose of this note is to review this development and describe our construction of this theory in the period 1982-85.
\end{abstract}

PACS numbers: 12.60.Fr, 12.10.Dm, 14.80.Ly

\footnotetext{
* Email:arnowitt@physics.tamu.edu

† Email:chams@aub.edu.lb

‡ Email:nath@neu.edu
} 


\section{INTRODUCTION}

Supergravity grand unification (SUGRA GUT) is currently the main framework in theory models for the exploration of new physics beyond the standard model of electroweak and strong interactions. The framework allows one to extrapolate data from the electroweak scale all the way up to the scale of grand unification $M_{G} \sim 2 \times 10^{16}$ $\mathrm{GeV}$ and vice versa to test new ideas for the unification of particles and forces. Thus supergravity grand unification provides the framework to test new data from the LHC in a broad class of models. Supergravity grand unification was proposed by the authors in 1982 [1] and a significant development of this field has occurred since that time led by the authors and by other researchers. Supergravity grand unification produces a number of theoretical insights into the high energy domain and it has had an important impact on the progress of high energy theory since its inception which includes providing the leading dark matter candidate, the neutralino. In the following we summarize the major accomplishments of the SUGRA GUT and indicate the key elements that were needed in the development of this model highlighting our contribution to the original proposal of this model in 1982 and its further development.

The construction of supergravity grand unification was non-trivial, requiring several key elements. First it was necessary to construct couplings of gauge multiplets (representing the gauge bosons), to an arbitrary number of chiral multiples (representing the quarks and leptons) interacting supersymmetrically among themselves and to supergravity. Since spontaneous breaking of supergravity requires a super Higgs field whose vacuum expectation value is of order of the Planck mass $\left(M_{P l}\right)$, it was necessary to introduce a hidden sector (where the super Higgs fields reside) separate from the visible sector (where the quarks, leptons, the electroweak Higgs and the gauge fields reside) with no direct interactions between the hidden and the visible sectors except gravitationally. Thus interactions are suppressed by factors of $1 / M_{P l}$ between the hidden and the visible sectors. Such a separation of visible and hidden sector protects the low energy theory from mass growths of size $O\left(M_{P l}\right)$. However, there was another important gauge 
hierarchy problem that needed to be resolved in supergravity grand unification due to the presence of a second heavy scale, i.e., the GUT scale $M_{G} \simeq 2 \times 10^{16} \mathrm{GeV}$. A priori one could imagine corrections to the mass squared of size $m_{s} M_{G}\left(\kappa M_{G}\right)^{n}$ for $n=0,1,2, \ldots ., 6$ (where $m_{s}$ is of electroweak size and $\kappa=1 / M_{P l}$ ) destroying the gauge hierarchy. Thus an important step in the construction of a supergravity grand unification required an explicit exhibition of the remarkable fact that the low energy theory was free of such terms.

The outline of the rest of the paper is as follows: In Sec. II we discuss global supersymmetry and the problems faced in constructing viable models based on global supersymmetry. In Sec. III we discuss the couplings of gauge multiplets to matter multiplets and to supergravity. In Sec. IV we discuss spontaneous breaking of supergravity and resolving the Planck scale hierarchy problem. In Sec. V we discuss resolving the GUT scale hierarchy problem. In Sec. VI we discuss the low energy effective theory that results when one integrates out the heavy fields and the breaking of $S U(2) \times U(1)$ induced by the soft breaking of supergravity occurs completing the construction of SUGRA GUTs. In Sec. VII we discuss the parameter space of soft breaking. Phenomenological implications of supergravity grand unification are briefly discussed in Sec. VIII including a brief discussion of the implications of the recent discovery of a low mass possible Higgs boson at $125 \mathrm{GeV}$. Further developments are discussed in Sec. IX.

\section{PROBLEMS OF MODEL BUILDING IN GLOBAL SUPERSYMMETRY}

By about 1980, the development of global supersymmetry theory (SUSY) (which first appeared in two dimensions[2] and then in four dimensions[3-6]) had reached a halting point. While supersymmetry could tame the gauge hierarchy problem [7-9], and achieve a reasonable grand unification of the $S U(3) \times S U(2) \times U(1)$ coupling constants $[10][8,9]$, it could do this only if supersymmetry was broken in order to grow the masses for the new SUSY particles. The only credible way of breaking SUSY would be through spontaneous breaking. However, spontaneous breaking of SUSY appeared to be very difficult. The SUSY Hamiltonian is positive semi-definite, and so the lowest energy state 
is the supersymmetric one with $E=0$, the broken states lying above. Further, if SUSY was not broken at the tree level, it did not break at the loop level $[11,12]$. Even if SUSY could be broken spontaneously, unsatisfactory phenomena arose, e. g. a mass spectrum in gross disagreement with experiment, and in violation of what was needed for the grand unification. In addition, the breaking of a global symmetry leads to a massless Goldstone particle, in this case a massless fermionic goldstino, that did not appear experimentally, as it was ruled out as a possible candidate for the neutrino. Thus while it was possible to exhibit the type of SUSY breaking terms that maintained the hierarchies, i.e. the so-called soft breaking terms [13], a reasonable model where such terms arose naturally and with experimentally acceptable values did not exist. Without a theoretical origin of the breaking of SUSY, one did not have a model that could be compared with experiment.

\section{COUPLING OF SUPERGRAVITY TO MATTER AND GAUGE MULTIPLETS}

It was as this point in 1981 that we turned to supergravity as a possible alternative. This was an area in which the interests of the three authors converged. Thus two of us (RA and PN) had worked on local supersymmetry $[14,15]$ which was a precursor of the current supergravity theory $[16,17]$ while the other author (AHC) who also worked on supergravity [18] was at that time actively engaged on the reduction of higher dimensional supergravity theories $[19,20]$. Further, by 1980 it was clear that nature embraced local gauge theory in both the Standard Model (SM) of strong and electro-weak interactions and also in gravity [21]. Thus using a local supersymmetry to build models was a natural idea. At that time, however, there were no phenomenological supergravity models of particle interactions. The reason for this was that the theory of how to couple an arbitrary matter content to supergravity did not exist. In general relativity, the coupling of matter to gravity is very simple: one just adds the matter Lagrangian, appropriately covariantized, to the Einstein Lagrangian to obtain minimal coupling. In supergravity things were much more complicated. A Lagrangian with one chiral multiplet coupled to supergravity had 
been constructed by Cremmer et al. in 1979 [22] using the rules of super-conformal tensor calculus $[23,24]$. However, what was needed in order to construct realistic models of nature was the coupling of an arbitrary number of chiral multiplets (representing quarks, leptons and Higgs particles) and gauge multiplets to supergravity, to describe the gauge interaction with the chiral multiplets and the gauge and chiral multiplets with the supergravity fields.

This was a very complicated task, which was completed by us by early Spring of 1982 using the rules of supersymmetric tensor calculus. It was found that the most general set of couplings involved a superpotential $W\left(Z_{A}\right)$, a Kähler potential $K\left(Z_{A}, Z_{A}^{\dagger}\right)$ and a gauge kinetic function $f_{A B}\left(Z_{A}\right)$ where $Z_{A}$ are the bose components of the chiral multiplets. The first two appeared in the Lagrangian in the combination [1, 25-27]

$$
G=-\kappa^{2} K\left(Z, Z^{\dagger}\right)-\ln \left(\frac{\kappa^{6}}{4}|W|^{2}\right)
$$

where $\kappa=1 / M_{P l}$, and $M_{P l}=2.4 \times 10^{18} \mathrm{GeV}$. The Lagrangian contained the scalar potential

$$
V=-\frac{1}{\kappa^{4}} e^{-G}\left(3+\left(G^{-1}\right)_{B}^{A} G_{, A} G,^{B}\right)+\frac{1}{2 \kappa^{4}} \operatorname{Re}\left(f^{-1}\right)_{r s} D^{r} D^{s}
$$

where $\left(G^{-1}\right)_{B}^{A}$ is the inverse of $G_{, A}^{B}$ and where $D^{r}=-g_{r} \kappa^{-2}\left(G_{i}\left(T^{r}\right)_{i j} Z_{j}\right)$ along with a complex array of terms involving gauge and fermionic interactions involving $f_{A B}$ and $G$. The potential $V$ reduces correctly to that of global SUSY in the limit of $M_{P l} \rightarrow \infty$, showing that one should be able to recapture the correct physics of the Standard Model and it's SUSY generalization in the low energy limit (as needed for any physically correct generalization).

However, the most exciting result was that due to the supergravitational corrections, the potential $V$ was no longer positive, and thus it was possible to spontaneously break supersymmetry as was first observed in [22] (where a single chiral multiplet was coupled to supergravity). In addition there was sufficient freedom to fine tune the cosmological constant to its physical value. This implied that the (super) gravitational corrections play a crucial role in setting up a viable supersymmetric theory. This if realized in nature would be a milestone in physical theory as it would be the first time that gravity 
impacted on particle physics in a fundamental and direct way.

\section{SPONTANEOUS BREAKING OF SUPERGRAVITY AND RESOLVING THE PLANCK SCALE HIERARCHY PROLBLEM}

Although we had all the results on $N=1$ supergravity coupled to an arbitrary number of chiral multiplets and to vector muliplets in early spring of 1982, we did not immediately publish them ${ }^{1}$ (The full details of that construction were given in lectures at ICTP in 1983 and published in the Trieste Lecture series [27]). Similarly in early spring of 1982 we had also obtained results on the breaking of a GUT group within a supergravity grand unification context. Here we observed a new phenomena. It was well known that the breaking of a GUT group in global supersymmetry led to degenerate vacua. However, in a supergravity grand unification we noticed that this degeneracy was broken. Specifically, if one of the vacua was chosen where the vacuum energy was arranged to vanish then the remaining vacua corresponded to lower vacuum energies. Again we did not immediately publish these results [29] and soon after we became aware that similar results had been obtained independently by Steven Weinberg[30]. (Weinberg had in addition shown that each of the resultant vacua were in fact stable against bubble formation and thus the physically desirable vacuum state with the $S U(3) \times S U(2) \times U(1)$ invariance was actually stable.)

The reason for not publishing our results both on the $N=1$ supergravity coupled to an arbitrary number of chiral multiplets and to vector multiplets and on the lifting of the degeneracy by gravitational effects of the various vacuum states after the GUT symmetry was broken, was because we were focused on the major theoretical issue of how to break supersymmetry and grow the necessary soft breaking terms. (The results on the lifting of the vacuum degeneracy were later mentioned in [1] and for a later work see also [31]). Again, the breaking would have to be spontaneous, e. g. by a super

\footnotetext{
${ }^{1}$ For previous reviews of the history see [28].
} 
Higgs effect, and a simple model that allowed for this was a linear term in the super potential [32]: $W=m^{2}(Z+B)$ where $\mathrm{Z}$ was the super Higgs field discussed earlier in [22]. Minimizing the potential $V$ gave $\kappa<Z>=O(1)$, and $B$ can be used to adjust the cosmological constant. We later realized that the precise form of the super Higgs potential was not crucial for the low energy theory provided it had the form $W=m^{2} f(\kappa Z) / \kappa$ where at the minimum $\kappa<Z>=O(1)$ so that $<W>=O\left(m^{2} / \kappa\right)$. This breaking of the supergravity gauge invariance then led to the growth of mass for the gravitino and the SUSY particles of size $\kappa^{2}<W>=\kappa m^{2}$. This told us two things: First, if we chose $m_{s} \equiv$ $\kappa m^{2}=O(T e V)$, i.e., $m \sim 10^{10} \mathrm{GeV}$, then the soft breaking masses would be in the right scale to get grand unification. Second since we were now breaking a local supersymmetry, the Goldstino problem was automatically solved, as it would be absorbed by growing mass for the gravitino (as was initially suggested by Volkov and Akulov[4] and worked out in detail in [22]).

The fact that in SUSY the gauge coupling constants unify at the GUT scale $M_{G}$ implies that one should construct a supergravity grand unified model (SUGRA GUT) where the GUT group breaks to $S U(3)_{C} \times S U(2)_{L} \times U(1)_{Y}$ at $M_{G}$ where $M_{G} \simeq 2 \times 10^{16} \mathrm{GeV}$. The main problem here was to see how to preserve the gauge hierarchy for both $M_{P l}$ and $M_{G}$ interactions. Let us decompose the set of fields $Z_{A}$ so that $Z_{A}=\left(Z, Z_{a}\right)$ where $Z$ as above is the super Higgs field and $Z_{a}$ are the set of visible sector fields. Thus a term in the superpotential of the form $Z Z_{a} Z_{b}$ would grow a Planck scale mass when $Z$ grows its $\mathrm{VEV}<Z>\sim M_{P l}$, unless the coupling is strongly suppressed (a possibility we will discuss later). We showed, however, that one may maintain the Planck scale hierarchy by assuming the superpotential to have the form [1]

$$
W_{t o t}=W\left(Z_{a}\right)+W_{S H}(Z)
$$

Here $W\left(Z_{a}\right)$ is the superpotential that governs the interactions of the chiral fields in the visible sector, i.e., quarks, leptons, and Higgs both light and heavy, and $W_{S H}(Z)$ is the superpotential in the hidden sector which breaks supersymmetry spontaneously. Now if gravity were absent, as is the case in global supersymmetry, the two sectors will be 
totally separate with no influence of one sector on another. However, as a consequence of the fact we are working in the framework of supergravity the effect of breaking in the hidden sector can be felt in the visible sector, via the supergravity coupling of the two sectors (see Eq.(2)). Thus the super Higgs lives in a "hidden" sector that communicates via the effective potential $\mathrm{V}$ only gravitationally. (This is now referred to as gravity mediated breaking.) The separation of the two sectors of Eq.(3) guarantees, however, that the low energy sector is protected against Planck scale mass growth by factors of $1 / M_{P l}$. This provided the first model where gravity plays a central role in particle physics.

\section{RESOLVING THE GUT SCALE HIERARCHY PROBLEM}

The separation of the hidden sector from the physical sector in Eq.(3) and the size of $m^{2}$ in $m_{s} \equiv \kappa m^{2}$ guarantees the absence of Planck scale corrections to the low energy masses. However, the nearness of the GUT scale to the Planck scale, leads to a new gauge hierarchy problem, since one might have mass $^{2}$ corrections to the visible sector fields of the type

$$
m_{s} M_{G}, m_{s} M_{G}\left(\kappa M_{G}\right), \cdots, m_{s} M_{G}\left(\kappa M_{G}\right)^{k},
$$

destroying the hierarchy for $k \leq 6$. To examine the GUT scale hierarchy, one may divide the visible sector fields as $Z_{a}=\left(Z_{\alpha}, Z_{i}\right)$ where $Z_{i}$ are the heavy fields and $Z_{\alpha}$ are the light fields.

To understand physically how the theory prevents the existence of corrections of the type in Eq.(4) we first note that the protection of the low energy mass from the Planck mass by having supergravity break in the hidden sector can be rephrased differentially (and slightly generalized) by requiring that the potential in the visible sector involves a hidden sector field dependence only in the combination $\bar{Z} \equiv \kappa Z$

$$
W=W\left(\bar{Z}, Z_{\alpha}, Z_{i}\right)
$$

where the only dependence of $Z$ in $W$ is in the combination $\bar{Z}$. These conditions are of course satisfied if the super Higgs alone lives in a hidden sector, but also allows coupling 
of the super Higgs to the physical sector provided that coupling is suppressed by factors of $\kappa=1 / M_{P l}$. To protect the low energy masses from GUT scale masses after the breaking of the grand unified gauge group, we impose a similar constraint isolating the electroweak scale from the GUT scale:

$$
W_{, \alpha i}=O\left(\kappa m^{2}\right) ; \quad W_{, \alpha \beta}=O\left(\kappa m^{2}\right)
$$

To show that these conditions are sufficient to protect the weak scale from the GUT scale is non-trivial (as there are now three mass scales in the system, i.e., $M_{P l}, M_{G}, m$ ) and we give now a brief summary of the results of [33]. In the above case all terms of the type $m_{s} M_{G}\left(\kappa M_{G}\right)^{k}$ either cancel or are absent. This was first shown in the specific model of [1], and later shown to hold for the more general class of SUGRA GUT models in [33]. An alternative derivation was given in [34].

The absence of $M_{G}$ from the soft breaking sector of the theory is central to setting up a supergravity grand unified model. In order to obtain the low energy Lagrangian in a GUT theory, i.e., at scales $E<M_{G}$, one must integrate out both the super Higgs fields as well as the GUT fields. This means in principle that one uses field equations

$$
\frac{\partial V}{\partial Z}=0, \quad \frac{\partial V}{\partial Z_{i}}=0
$$

to solve for the super Higgs field $Z$ and for the superheavy field $Z_{i}$ in terms of the light fields $Z_{\alpha}$. To implement this procedure we expand the VEVs of the super Higgs field, of the heavy fields and of the light fields in powers of $\kappa$ so that

$$
\begin{array}{r}
Z=Z^{-1}+Z^{(0)}+\cdots \\
Z_{i}=Z_{i}^{(0)}+Z_{i}^{(1)}+Z_{i}^{(2)}+\cdots \\
Z_{\alpha}=Z_{\alpha}^{(1)}+Z_{\alpha}^{(2)}+\ldots,
\end{array}
$$

where $Z^{(n)}$ is of order $\kappa^{n}$. Such a solution, in general, would involve the GUT scale $M_{G}$ and one needs to show that the low energy potential $V_{\text {eff }}$ defined by the above elimination process, i.e., 


$$
V_{e f f}\left(Z_{\alpha}\right)=V\left[Z_{i}\left(Z_{\alpha}\right) ; Z_{\alpha} ; Z\left(Z_{\alpha}\right)\right]
$$

is independent of the scale $M_{G}$. A procedure similar to this was followed in [34]. The route followed in the analysis of $[1,33]$ was to examine directly the field equations for the light modes where the extrema constraints arising from the super Higgs and the heavy fields, i.e., the constraints of Eq.(7) have already been imposed and then show that the resulting equations could be obtained by varying an effective potential $V_{\text {eff }}$, and that $V_{\text {eff }}$ to leading order $m_{s}^{4}$ was independent of $M_{G}$.

To see the complexity of the problem one can consider the field equations for $Z_{A}$ (obtained by varying Eq.(2)) which take the form

$$
T_{A B} G_{B}=0 .
$$

Here $G_{A}$ is given by

$$
G_{A}=W_{, A}+\frac{1}{2} \kappa^{2} Z_{A}^{\dagger} W
$$

and $T_{A B}$ by

$$
T_{A B}=W_{, A B}+\frac{1}{2} \kappa^{2}\left(Z_{A} G_{B}+Z_{B} G_{A}\right)-\frac{1}{4} \kappa^{4} Z_{A} Z_{B} W-\kappa^{2} \delta_{A B} W,
$$

where $W_{, A} \equiv \partial W / \partial Z_{A}$. In writing these quantities it is convenient to rescale the variables appearing as follows

$$
Z_{\alpha}=m_{s} z_{\alpha}, \quad Z_{i}=M_{G} z_{i}, \quad Z=M_{P l} z
$$

and further rescalings so that

$$
W=m^{2} M_{P l} \bar{W}, \quad G_{\alpha}=m_{s}^{2} \bar{G}_{\alpha}, G_{i}=m_{s}^{2} \bar{G}_{i}, \quad G_{Z}=m^{2} \bar{G}_{Z}
$$

where all the barred quantities are dimensionless. The three equations of Eq.(10) for $A=Z, Z_{i}, Z_{\alpha}$ then take the form

$$
\begin{array}{r}
{\left[\frac{1}{m_{s}} W_{S H, Z Z}+\left(z \bar{G}_{Z}-\bar{W}-\frac{1}{4} z^{2} \bar{W}\right)+\frac{1}{2}\left(\epsilon \delta_{s} z_{i} \bar{G}_{i}+\delta_{s}^{2} z_{\alpha} \bar{G}_{\alpha}\right)\right] \bar{G}_{Z}} \\
+\frac{1}{4} \epsilon \delta_{s} z z_{i} \bar{G}_{i} \bar{W}+\frac{1}{2} \delta_{S}^{2}\left(z \bar{G}_{i}^{2}+z \bar{G}_{\alpha}^{2}-\frac{1}{2} z z_{\alpha} \bar{G}_{\alpha} \bar{W}\right)=0,
\end{array}
$$




$$
\begin{array}{r}
{\left[W_{, i j}+\frac{M_{G}}{2}\left\{\delta_{s}^{2}\left(z_{i} \bar{G}_{j}+z_{j} \bar{G}_{i}\right)-\frac{1}{2} \epsilon \delta_{s} z_{i} z_{j} \bar{W}\right\}\right.} \\
\left.+m_{s} \delta_{i j}\left\{\frac{1}{2} z \bar{G}_{z}-\bar{W}+\frac{1}{2} \delta_{s}^{2} z_{\alpha} \bar{G}_{\alpha}\right\}\right] \bar{G}_{j} \\
+M_{G}\left[\frac{1}{2} z_{i} \bar{G}_{Z}^{2}-\frac{1}{4} z_{i} z \overline{W G}_{Z}+\frac{1}{2} \delta_{s}^{2} z_{i}\left(\bar{G}_{\alpha}^{2}-\frac{1}{2} \bar{G}_{\alpha} \bar{W}\right)\right]=0,
\end{array}
$$

and

$$
\begin{gathered}
\left(\frac{1}{m_{s}} W_{, \alpha \beta}+\delta_{\alpha \beta}\left(\frac{1}{2} z \bar{G}_{Z}-\bar{W}\right)+\frac{1}{2} \epsilon \delta_{s} \delta_{\alpha \beta} z_{i} \bar{G}_{i}+\frac{1}{2} \delta_{s}^{2}\left(z_{\alpha} \bar{G}_{\beta}+z_{\beta} \bar{G}_{\alpha}\right)-\frac{1}{4} z_{\alpha} z_{\beta} \bar{W}\right) \bar{G}_{\beta} \\
+\left(\bar{W}_{, \alpha i} \bar{G}_{i}+\frac{1}{2} z_{\alpha} \bar{G}_{Z}^{2}-\frac{1}{4} z_{\alpha} z \bar{W} \bar{G}_{z}\right)-\frac{1}{4} \epsilon \delta_{s} z_{\alpha} z_{i} \bar{W} \bar{G}_{i}+\frac{1}{2} \delta_{s}^{2} z_{\alpha} \bar{G}_{i}^{2}=0 .
\end{gathered}
$$

Eqs. $(15,16,17)$ contain two parameters of smallness

$$
\epsilon=\kappa M_{G} \sim 10^{-2} ; \quad \delta_{s}=\kappa m_{s} \sim 10^{-16}
$$

(The work of [34] does not distinguish between $M_{P l}$ and $M_{G}$, and assumes a large common mass $M$ for them.) Since $W_{, i j}$ is proportional to the heavy sector mass matrix, i.e., $W_{, i j} \sim M_{G}$, we see that Eq.(6) allows solutions of Eqs.(15, 16, 17) where all the dimensionless barred and lower case fields are $O(1)$ (with corrections of size $\epsilon \delta_{s}$ and $\delta_{s}^{2}$ ). This is remarkable since on dimensional grounds one would have expected $G_{i} \sim M_{G}^{2}$. Eqs.(15) and (16) allow one to determine $Z$ and $Z_{i}$ in terms of $Z_{\alpha}$ and when inserted into Eq.(17) gives the equation to determine $z_{\alpha}$ which is then independent of $M_{G}$ (aside from small $\epsilon \delta_{s}, \delta_{s}^{2}$ corrections). After a detailed analysis, it is shown that the resultant Eq.(17) is the field equation deducible from varying an effective potential $V_{\text {eff }}$ depending only on the low energy fields $Z_{\alpha}[33]$.

The above discussion exhibits several interesting features of the SUGRA GUT models: (i) As can be seen from Eq.(17) the supergravity interactions do produce additional couplings between the GUT scale and the Planck scale fields and the low mass section of the theory which are not found in global supersymmetry, but these pieces do not add large 
(i.e., order $M_{G}$ or $M_{P l}$ ) corrections to the size of the low mass fields and thus maintain the gauge hierarchy.

(ii) The above equations allows one to calculate the size of the corrections to the low energy spectrum from $M_{G}$ and $M_{P l}$. From Eq.(17) one sees that they are of size $\epsilon \delta_{s}$ and $\delta_{s}^{2}$ and hence very small. Thus the theory strongly protects the electroweak scale physics from high scale physics, which justifies using the electroweak SUSY mass spectrum up to the GUT scale, as required by the renormalization group analysis which ranges from the GUT scale down to the electroweak scale.

(iii) The constraints Eq.(6) put certain restrictions on the type of superpotential one may allow. Thus the coupling of two light fields and one heavy field such as $c_{\alpha \beta i} Z_{\alpha} Z_{\beta} Z_{i}$ is allowed since $W_{, \alpha i}$ in this case will be proportional to a $Z_{\beta}$ with $\operatorname{VEV~} O\left(m_{s}\right)$ which satisfies the constraint of Eq.(6). (The second condition of Eq.(6) is satisfied provided the VEV of $Z_{i}$ vanishes.) Similarly, the coupling of a light field to two heavy fields such as $c_{\alpha i j} Z_{\alpha} Z_{i} Z_{j}$ is forbidden unless the $\mathrm{VEVs}$ of $Z_{i}$ and $Z_{j}$ vanish since $W_{, \alpha i}$ in this case will be proportional to $Z_{j}$. (However, such heavy-heavy-light couplings were later shown to destabilize the gauge hierarchy at the loop level if $Z_{\alpha}$ also had couplings to light particles $[35-37]$.

\section{LOW ENERGY EFFECTIVE THEORY AND $S U(2) \times U(1)$ BREAKING}

With these results the effective potential of the low energy theory takes on a remarkably simple form. For the case $f_{\alpha \beta}=\delta_{\alpha \beta}$ and a flavor blind Kahler potential one has

$$
V_{e f f}=\left|\frac{\partial \tilde{W}}{\partial Z_{\alpha}}\right|^{2}+m_{0}^{2} Z_{\alpha}^{\dagger} Z_{\alpha}+\left(B_{0} W^{(2)}+A_{0} W^{(3)}+h . c\right)+\frac{1}{2}\left[g_{\sigma} \kappa^{-2}\left(G_{\alpha}\left(T^{\sigma} Z\right)_{\alpha}\right)\right]^{2},
$$

where $\tilde{W}$ is the superpotential containing only quadratic and cubic functions of the light fields, i.e., $\tilde{W}\left(Z_{\alpha}\right)=W^{(2)}\left(Z_{\alpha}\right)+W^{(3)}\left(Z_{\alpha}\right)$, and $m_{0}, A_{0}, B_{0}$ are soft breaking parameters of size $m_{s}$.

The proof of the absence of $M_{G}$ from the low energy theory after spontaneous breaking of supersymmetry and of the GUT symmetry for a specific GUT group was completed in early summer of 1982 and given in [1] (submitted July 12). (The extension to the 
general class of grand unified models was subsequently made in [33, 34].). Thus Ref. [1], published in 1982, represents the first construction of a viable supergravity GUT model.

It is interesting to note that Eq.(19) (along with gaugino masses discussed below) represent the first phenomenologically viable soft terms arising from spontaneous breaking of supersymmetry. Thus the spontaneous breaking of supergravity at the Planck scale gives rise naturally to the soft breaking needed phenomenologically. The model of Ref. [1] assumed, for simplicity, an SU(5) GUT group, a flat Kahler potential, and a gauge kinetic function $f_{\alpha \beta}=\delta_{\alpha \beta}$. The breaking of the Standard Model gauge group to $S U(3)_{C} \times U(1)_{E M}$ was accomplished there at the tree level by introduction of a singlet field $U$ with coupling $U H_{1} H_{2}$. Soft breaking masses for squarks, sleptons and gauginos occurred and their phenomenologies were investigated [38-44]. Initially the gaugino masses were generated at the loop level [41, 45-49] by the exchange of heavy fields but a more direct way of producing them exists by giving the gauge kinetic energy function a non-trivial field dependence $[25,26]$.

The most remarkable feature, however, was that it was the breaking of supergravity in the hidden sector that caused the breaking of $S U(2)_{L} \times U(1)_{Y}$. The above tree level breaking of $S U(2)_{L} \times U(1)_{Y}$ was later seen to be unstable at the loop level $[35,36]$ and a theoretically more desirable model of breaking $S U(2)_{L} \times U(1)_{Y}$ could be realized using the renormalization group equations (RGE). Thus the running of the RGE from the GUT scale to the electro-weak scale drives one of the eigenvalues of the Higgs (mass) ${ }^{2}$ matrix negative causing the electro-weak symmetry breaking at this scale [45]. Again it is the presence of the soft terms arising from the spontaneous breaking of supergravity that leads to the breaking of $S U(2)_{L} \times U(1)_{Y}$. (For early work within global supersymmetry see [50, 51] though here there was no theoretical deduction of soft breaking masses available to realize the possibility.). The origin of this remarkable effect is the largeness of the top quark Yukawa coupling (i.e., a large top mass). This is what drives the soft breaking of up quark Higgs (mass) ${ }^{2}$ (generated by the breaking of supergravity in the hidden sector) downwards as we move from the GUT scale down to the electroweak scale. 
The fact that the super Higgs mass scale, $m_{s}=\kappa m^{2}$, of the soft breaking parameters and the scale of $S U(2) \times U 1)$ breaking are comparable, i.e., both lie in the $\mathrm{TeV}$ region, is a natural consequence of the heavy top quark. In fact, SUGRA GUTs was the only model in the early eighties that predicted the top quark to be heavy i. e. $\gtrsim 100 \mathrm{GeV}$ [44-48]. Most significant is the fact that the unnatural negative Higgs boson (mass) ${ }^{2}$ term needed in the Standard Model to generate spontaneous breaking of the electroweak symmetry (and grow masses for the $\mathrm{W}$ and $\mathrm{Z}$ gauge bosons and the quarks and leptons) occurs in SUGRA GUTs in a natural way.

During this period, a number of other researchers, who made interesting and important contributions, were engaged in examining supergravity theory and we conclude this section with a brief discussion of the relation between these papers and our work. The work of Cremmer, Ferrara, Giradello and van Proeyen [25] (submitted June 9, and published October 14) was concerned with the construction of the coupling of chiral and gauge multiplets to supergravity and the super Higgs effect. As discussed above we had completed this analysis ourselves in early Spring 1982, but did not immediately publish it as we were most interested in how to use this to construct a grand unified model. (Our work on the supergravity couplings was later published in [27]) These authors also generalized the supertrace formula, and showed that if $m<<\left(M_{W} M_{\mathrm{Pl}}\right)^{1 / 2}$ supergravity contributions were negligible, while when $m>\left(M_{W} M_{\mathrm{Pl}}\right)^{1 / 2}$ supergravity was important and a reasonable tree level scalar spectrum was obtained. However, they did not attempt to build any physical models based on this. While this work was submitted prior to our Physical Review Letter[1] (July 12), we were unaware of it as we did not receive a preprint from the authors before our paper was written. It is also clear that we could not have written our July 12 paper without having worked out the details of the supergravity couplings independently.

The work of Barbieri, Ferrara and Savoy [52] (BFS) was submitted August 3 and so post dates our paper (July12). Further the authors were already aware of our work 
through the preprint sent earlier to Ferrara [The preprint distributed at that time contained minor errors in the form of the $\mathrm{D}$ term in the Lagrangian and the extrema of the superHiggs field. Neither of these effected the results in the paper, and the published version in Physical Review Letters[1] contains no errors.]. The work of BFS makes use of the Cremmer et al. 82 paper [25] to couple low energy global SUSY models to supergravity. Thus no GUT theory is discussed. Further, the electroweak breaking is not of super gravitational origin as it is anchored in the global supersymmetric Fayet model [53]. Thus the model is not really a simplified version of a GUT model. The BFS paper, however, was of theoretical interest, as it introduces the technique of eliminating the superHiggs in the Lagrangian in terms of its VEV and the low energy fields, which exhibits clearly the origin of the soft breaking terms, a technique which has been found useful in later works.

As discussed briefly before, the presence of a $U H H^{\prime}$ term in the super potential (where $U$ is a singlet and $H$ and $H^{\prime}$ are 5 and $\overline{5}$ representations of $\mathrm{SU}(5)$ ) requires a large fine tuning to control loop corrections [35, 36]. The work of Ferrara, Nanopoulos and Savoy [54] (submitted December 20) was a useful addition to the literature on the subject at that time in that it examined the possibility of evading this problem by using more complicated SU(5) representations, i. e. 75, 50 and $\overline{50}$. These authors found that by assuming various possible partial symmetries in the superpotential, one could indeed suppress the loop corrections by using (technically natural) fine tunings. Thus models do exist where the loop problem can be controlled. However, the fact that the top quark turned out to be very heavy, has led to loss of interest in such approaches, and the general adoption of using the more elegant renormalization group approach described above, where the breaking of supersymmetry at the high scale by the superHiggs effect causes the breaking of $S U(2) \times U(1)$ at the electroweak scale, by naturally turning one of the eigenvalues of the Higgs mass ${ }^{2}$ matrix negative at the electroweak scale.

We conclude this brief discussion of other work at the time of our paper [1] with the 
paper of Ibanez [55] (submitted August 3, revised August 24). This paper appeared after ours (July 12), and the author references our work and so was aware of it. This is the only other work at that time aside from our paper [1] which attempts to build a full supergravity grand unification model (though no model is fully worked out and the low energy limit of the models considered is not examined). Further, the models that the author considers are left, at the minimum of the effective Lagrangian, with a large negative cosmological constant, and the suggestion made to eliminate it in the Lagrangian by hand, thus breaking supersymmetry by hand. We believe that such models are basically unsatisfactory, and like the suggestions of [56] in fact have not been pursued further. However, the author does stress the importance of radiative corrections in going from the high energy scale down to the electroweak scale. The subsequent (1983) work by Ibanez and colleagues [46-48] along with the work of Alvarez-Gaume, Polchinski and Wise [45] using the renormalization group approach for the mSUGRA model (of $\mathrm{Eq}(20)$ below) made an important contribution to understanding how one may detect SUGRA particles at accelerators.

\section{SOURCES OF SOFT BREAKING AND THE PARAMETER SPACE OF SUGRA GUTS}

While global supersymmetry can accommodate over 100 soft breaking parameters (since there is no theory as to their origin in global supersymmetry), SUGRA GUTs allows one to build simple models that are relatively natural and with a significantly reduced number of soft terms. As mentioned already loop corrections can give rise to universal gaugino masses $[41,45,49]$. Gaugino masses can also be obtained at the tree level by including a field dependence in $f_{\alpha \beta}$ to read $\delta_{\alpha \beta}+\kappa c_{\alpha \beta Z} Z$ where $c_{\alpha \beta Z}$ is dimensionless $O(1)[25,55]$.

The simple example considered above and in [1] assumed a flat Kahler potential,

$K=\sum_{A} Z_{A} Z_{A}^{\dagger}$ and implied a relation between $A_{0}$ and $B_{0}$. However, the extension to a more general Kahler potential is straightforward. Thus one may choose a non-flat but still flavor conserving Kahler potential or a more general form of the Kahler potential allowing for couplings between the hidden and the visible sectors but suppressed by the Planck mass, releasing the constraint between $A_{0}$ and $B_{0}$. Thus one might add terms 
to the superpotential where the hidden sector field always occurs in the combination $\kappa Z$. Since $\kappa<Z>\sim O(1)$ the gauge hierarchy will be preserved when a term of this type multiplies superpotential terms in the visible sector [57]. (This is an example of violating the principle of separation of visible and hidden sectors, which, however, is acceptable here on phenomenological grounds since the couplings are suppressed by the factor $\kappa=1 / M_{P l}$.) Thus consider a superpotential where couplings have dependence on the hidden sector fields, so that $W=W^{(2)}+W^{(3)}$ where $W^{(2)}=f_{2}(\kappa Z) g^{(2)}\left(Z_{\alpha}\right)$ and $W^{(3)}=f_{3}(\kappa Z) g^{(3)}\left(Z_{\alpha}\right)$ where $Z$ as before is the hidden sector field and $g^{(2)}\left(g^{(3)}\right)$ are quadratic (cubic) in the visible sector fields $Z_{\alpha}$. A direct computation then shows that cross terms between $W$ and $W_{S H}$ in the scalar potential give additional contributions to dimension 2 and dimension 3 soft operators. With the additional term one finds $\Delta V_{\text {eff }}=\Delta B_{0} W^{(2)}+\Delta A_{0} W^{(3)}+$ h.c. where $\Delta B_{0}=\bar{m}_{s}\left(\ln f_{2}\right)^{\prime}$ and $\Delta A_{0}=\bar{m}_{s}\left(\ln f_{3}\right)^{\prime}$ where $\bar{m}_{s}=e^{\kappa^{2} Z Z^{*}} \kappa^{2}<W_{S H}>\kappa<Z>$. Note that $\Delta B_{0}$ and $\Delta A_{0}$ are uncorrelated since $f_{2}(\kappa Z)$ and $f_{3}(\kappa Z)$ are arbitrary functions. Initially it was seen that a cubic term of the type $U H_{1} H_{2}$ leads to a bilinear term of the type $\mu_{0} H_{1} H_{2}$ after the light singlet $U$ develops a VEV, and further $\mu_{0}$ is of size $\kappa m^{2}[1]$. A more satisfactory derivation of such a term was later shown to arise by a Kähler transformation with a holomorphic quadratic term of the type $\mathrm{cH}_{1} H_{2}+$ h.c. in the Kähler potential $[57,58]$. Thus one is led to a simple model with five universal parameters at the GUT scale: $m_{0}, m_{1 / 2}, A_{0}, B_{0}, \mu_{0}$. These parameters characterize the way the super Higgs field interacts with the matter fields. More significantly since they arise from $\kappa=1 / M_{P l}$ corrections, they give information on Planck scale physics. Models of this type offer the possibility of understanding the origin of different mass scales.

After minimizing the effective potential at the electro-weak scale, one is left with four parameters and one sign:

$$
m_{0}, A_{0}, m_{1 / 2}, \tan \beta=<H_{2}>/<H_{1}>, \operatorname{sign}(\mu) .
$$

The model above has been called variously: supergravity grand unified model[59], minimal supergravity model[60], CMSSM[61] or mSUGRA [62] The mSUGRA model ${ }^{2}$ is

\footnotetext{
${ }^{2}$ Recently, a few people have used the notation mSUGRA to mean the special model where $B_{0}=A_{0}-m_{0}$
} 
particularly simple and as a consequence of that it rapidly became a benchmark for the investigation of the effects of SUSY models on various phenomena.

As noted in the beginning of this section a flat Kahler potential will lead to a relation between $A_{0}$ and $B_{0}$. However, a general Kahler potential which is still flavor blind relaxes this relation and results in Eq.(20). Using the parameter space of Eq.(20) the mass spectrum of the superpartners of the quarks, leptons and gauge bosons were computed and further, the low energy supersymmetric Lagrangian in the mass diagonal states was worked out $[38,39,41,42]$. Specifically the chargino and neutralino mass eigenstates were exhibited to be linear combinations of gauginos and higgsinos which were found to lead to a rich low energy phenomenology controlled by the relative strength of the gaugino and higgsino components in the mass eigenstates. It was also noted that the decays of the sparticles will lead to events with missing energy in models with $\mathrm{R}$ parity conservation (for a review of the early work on the phenomenological applications of supergravity models see [63]).

\section{PHENOMENOLOGICAL APPLICATIONS OF SUGRA GUTS}

Having completed the discussion of the discovery of SUGRA GUT we now give a brief summary of some of the early subsequent developments that occurred. Thus an early applications was the computation of the supersymmetric loop contribution to the anomalous magnetic moment of the muon. It was found that the supersymmetric contribution could produce corrections which could be as large or larger than the Standard Model contribution $[64,65]$. This work in part provided the impetus for mounting the Brookhaven experiment E821. Current data gives $a_{\mu}^{e x p}-a_{\mu}^{S M} \simeq(2.87 \pm 0.8) \times 10^{-9}(\sim 3.5 \sigma)$ using $e^{+} e^{-}$annihilation for hadronic corrections and using $\tau$ data one has $a_{\mu}^{e x p}-a_{\mu}^{S M} \simeq(1.95 \pm 0.83) \times 10^{-9}$ $(\sim 2.4 \sigma)$ deviation from the Standard Model $[66,67]$. More recent analyses indicate that

(thus determining $\tan \beta$ after minimizing the effective potential). We view this as unfortunate usage as the acronym mSUGRA is clearly defined [62] and has been universally used in the literature since to mean the general model of $\mathrm{Eq}(20)$. 
supersymmetric contributions at two loop order can introduce a correction on the order of $10 \times 10^{-10} \quad$ [68].

Another early application of the supergravity models to electroweak loop phenomena was the computation of the supersymmetric electroweak corrections to the $\rho$ parameter defined by $\rho=M_{W}^{2} /\left(M_{Z}^{2} \cos ^{2} \theta_{W}\right)$ [69]. Corrections to this parameter are currently used to constrain supersymmetric models. As appropriate for a rapidly developing field, a number of further developments took place soon after including the formulation of noscale supergravity GUT models [70], and several reviews appeared subsequent to the fast moving developments in the period 1982-84, e.g. [71, 72].

As discussed at length above while the soft operators of dimensionality $d<4$ in the low energy theory are independent of the GUT scale, the dimension 5 or higher operator are very sensitive to this scale. Specifically the dimension five operators control the supersymmetric contribution to proton decay. Such dimension five operators arise in a supersymmetric GUT theory from the elimination of the heavy higgsino triplets which give

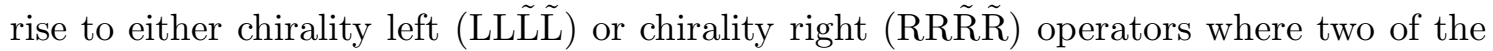
chiral left fields or chiral right fields are for sfermions denoted by the tilde on top. These operators when dressed by the exchange of chargino, neutralino and gluino exchanges produce baryon and lepton number violating dimension six operators involving four fermions with chiralities of the type LLLL, LLRR, RRRR, RRLL and lead to proton decay [73, 74].

While early analyses on the estimates of the proton lifetime existed $[75,76]$, the supergravity Lagrangian for baryon and lepton number violating operators for dimension five operators and their dressing with the full set of charginos, neutralinos and gluinos were first computed in $[77,78]$. This work represented the first complete analysis of proton decay lifetime for an $S U(5)$ model exhibiting further both the low scale and as well as the high scale implications of a supergravity unified model. Several new features emerged from this analysis including the importance of the L-R mixing effects and thus of the LLRR and RRLL as well as of RRRR operators after the dressings, and the possibility of cancellations among the dressing loop contributions from the first two generations and the third generation. Also a systematic analysis of a number of other decays modes aside 
from the dominant $\bar{\nu} K^{+}$mode such as $\bar{\nu} \pi^{+}, \mu^{+} \pi^{0}$, and $\mu^{+} K^{0}$ was given. These works are now standard references for comparison with experiment (see, e.g., [79, 80]).

Another important early implication of the supergravity unified model was the observation by Goldberg [81-83] that models with R-parity invariance possessed a natural candidate for the astronomically observed dark matter if the lightest supersymmetric particle was neutral, specifically the neutralino. Later it was shown using renormalization group analyses that over most of the parameter space of the mSUGRA model the lightest neutralino was indeed the lightest supersymmetric particle. Further, detailed calculations showed the remarkable result that the amount of dark matter remaining after freezeout was of the size that was seen astronomically. As a consequence of this, SUGRA GUT allows one to build a model of nature ranging from the low energy electroweak domain up to the GUT scale of $10^{16} \mathrm{GeV}$, and the prediction of cold dark matter within this model allows one to extrapolate backward in time to the freeze out at $10^{-8}$ sec. after the Big Bang. Thus the model allows for a remarkable unification of particle physics with early universe cosmology. The Large Hadron Collider can test the mSUGRA model by a study of the lepton and jet signatures and missing energy (For a recent discussion see [84]). Models of this type also allow measurements of neutralino production at the LHC and using the measurements only at the LHC predict[85] the amount of dark matter measured by Wilkinson Microwave Anisotropy Probe (WMAP) [86-88] and the Planck Satellite experiment [89]. The models also predict what properties might be seen by the direct detection of local Milky Way dark matter in experiments such as the Cryogenic Dark Matter Search (CDMS) [90, 91] and the XENON100 [92].

Another early application was in the analysis of supersymmetric signals under the assumption of $\mathrm{R}$ parity conservation. Thus with $\mathrm{R}$ parity, the decays of supersymmetric particles contain the LSP in its final products. If the LSP was a neutral particle, such as the lightest neutralino, one will have missing energy signatures in this case. One of the signatures that was discussed in this context was the trileptonic one [38-42] where, for example, a chargino plus the second lightest neutralino $\left(\tilde{\chi}_{2}^{0}\right)$ would have decays such 
as $\tilde{\chi}_{1}^{-} \rightarrow l^{-} \bar{\nu} \tilde{\chi}_{1}^{0}$ and $\tilde{\chi}_{2}^{0} \rightarrow l^{+} l^{-} \tilde{\chi}_{1}^{0}$ which would lead to a trileptonic signal and there was further work on it later on in $[93,94]$. These analyses were for on -shell decays of the $\mathrm{W}$ boson, i.e., $W \rightarrow \tilde{\chi}_{1}^{ \pm} \tilde{\chi}_{2}^{0}$ with further on-shell decays of $\tilde{\chi}_{1}^{ \pm}$and $\tilde{\chi}_{2}^{0}$. However, it was pointed out in [95] that the reach for discovering a chargino can be significantly extended if one considers off -shell decays of the $W$ boson, and most modern analyses utilize this feature in the analysis of the trileptonic signal $[96,97]$. In addition to the above a variety of signatures for supersymmetry were discussed in the 1980'e and 1990's. These include analyses for the production and decay of the gluino, the squarks and for the heavy Higgs bosons that arise in SUGRA models. The decays of the gluino and the squarks involve cascade decays. Thus, for example, gluinos which may be produced at the hadron colliders by either gluon fusion or quark fusion will undergo a decay chain such $\tilde{g} \rightarrow \tilde{q} \bar{q} \rightarrow \tilde{\chi}_{i}^{0} q \bar{q}, \tilde{\chi}_{k}^{ \pm} q_{1} \bar{q}_{2}$ etc while the squarks may also have cascade decays such as $\tilde{q} \rightarrow \tilde{g} q$ where $\tilde{g}$ decays as indicate previously. Such decays lead to multi-jet signatures, often accompanied by charged leptons, with missing energy. More detailed discussion of such cascade decays can be found in the literature [27, 98-100]. In the early 1990's the precision measurements of the grand unification of the gauge coupling constants consistent with supersymmetric grand unification simulated a major interest in SUGRA GUT and as mentioned earlier there were a number of works [101],[59? -61] which discussed the sparticle spectrum and other low energy supersymmetric phenomena and further work along these lines continues to this day.

With the coming on line of the LHC, the parameter space of SUGRA GUT models and specifically of mSUGRA is being constrained[102, 103]. More recently, LHC has found evidence of a possible Higgs boson with mass around $125 \mathrm{GeV}[104,105]$. This mass is consistent with mSUGRA which predicts a mass range for the Higgs boson to lie

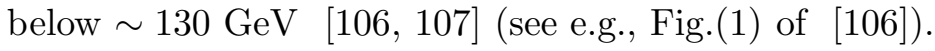




\section{FURTHER DEVELOPMENTS}

Of course mSUGRA is just the simplest of the SUGRA GUT models and there are supergravity models with non-universalities such as with non-universal gaugino masses at the GUT scale, and Higgs masses different from squark/slepton masses at the GUT scale. These can arise from non-universal choices for $f_{\alpha \beta}$ and $K[57]$. Which, if any, of these models are correct is a question that will hopefully be settled at the LHC. However, the various SUGRA models whether with universal or with non-universal soft breaking are simply variations on the supergravity grand unifications theme first proposed in [1]. If SUGRA GUT gets experimental support, that would impart an impetus for a fully unified theory within the string framework. This is so since string theory is seen to reduce to SUGRA GUT models at energies below the Planck scale [108]. Also the concept of a hidden sector first proposed in supergravity models could be identified with one of the $E_{8}$ (where supersymmetry is broken) in the $E_{8} \times E_{8}$ heterotic string theory and gravity mediation is often the mechanism utilized for the generation of soft masses. Thus SUGRA GUT models may be a way station on the road to a more complete unified theory.

Acknowledgments: This research work is supported in part by the U.S. National Science Foundation (NSF) grants PHY-070467, PHY-0757959 and PHY-0854779.

[1] A. H. Chamseddine, R. L. Arnowitt and P. Nath, Phys. Rev. Lett. 49, 970 (1982).

[2] P. Ramond, Phys. Rev. D 3, 2415 (1971).

[3] Yu A. Golfand and E.P. Likhtman, JETP Lett. 13, 323 (1971).

[4] D. Volkov and V.P. Akulov, JETP Lett. 16, 438 (1972).

[5] J. Wess and B. Zumino, Nucl. Phys. B78, 1 (1974).

[6] J. Wess, B. Zumino, Nucl. Phys. B70, 39-50 (1974).

[7] E. Witten, Nucl. Phys. B188, 513 (1981).

[8] S. Dimopoulos and H. Georgi, Nucl. Phys. B193, 150 (1981).

[9] N. Sakai, Zeit. f. Phys. C11, 153 (1981).

[10] S. Dimopoulos, S. Raby and F. Wilczek, Phys. Rev. D 24, 1681 (1981). 
[11] S. Weinberg, Phys. Lett. B62, 111 (1976).

[12] E. Witten, Nucl. Phys. B202, 253 (1982).

[13] L. Giradello and M. T. Grisaru, Nucl. Phys. B194 65 (1982).

[14] P. Nath and R. L. Arnowitt, Phys. Lett. B 56, 177 (1975);

[15] R. L. Arnowitt, P. Nath and B. Zumino, Phys. Lett. B 56, 81 (1975).

[16] D. Freedman, S. Ferrara and P. van Nieuwenhuizen, Phys. Rev. D13, 3214 (1976).

[17] S. Deser and B. Zumino, Phys. Lett. B62, 335 (1976).

[18] A. H. Chamseddine and P. C. West, Nucl. Phys. B129, 39 (1977).

[19] A. H. Chamseddine, Nucl. Phys. B185, 403 (1981);

[20] A. H. Chamseddine, Phys. Rev. D24, 3065 (1981).

[21] R. L. Arnowitt, S. Deser, C. W. Misner, in "Gravitation, an Introduction to Current Research", ed. L. Witten, J. Wiley, New York (1962). [gr-qc/0405109].

[22] E. Cremmer, B. Julia, J. Scherk, S. Ferrara, L. Girardello, P. van Nieuwenhuizen, Nucl. Phys. B147, 105 (1979).

[23] S. Ferrara, P. van Nieuwenhuizen, Phys. Lett. B76, 404 (1978).

[24] K. S. Stelle, P. C. West, Phys. Lett. B77, 376 (1978).

[25] E. Cremmer, S. Ferrara, L. Girardello, A. Van Proeyen, Phys. Lett. B 116, 231 (1982).

[26] E. Cremmer, S. Ferrara, L. Girardello, A. Van Proeyen, Nucl. Phys. B212, 413 (1983).

[27] P. Nath, R. L. Arnowitt and A. H. Chamseddine "Applied N=1 Supergravity," World Sci., Singapore. and Trieste Particle Phys.1983:1 (QCD161:W626:1983).

[28] A. H. Chamseddine, R. L. Arnowitt and P. Nath, Nucl. Phys. Proc. Suppl. 101, 145 (2001) [hep-ph/0102286].

[29] P. Nath, R. L. Arnowitt and A. H. Chamseddine, Phys. Lett. B 121, 33 (1983).

[30] S. Weinberg, Phys. Rev. Lett. 48, 1776 (1982).

[31] B. A. Ovrut and J. Wess, Phys. Lett. B 119, 105 (1982).

[32] J .Polonyi, University of Budapest Report No. FKI-1977-93, 1977 (unpublished).

[33] P. Nath, R. L. Arnowitt and A. H. Chamseddine, Nucl. Phys. B 227, 121 (1983).

[34] L. Hall, J. Lykken and S. Weinberg, Phys. Rev. D27, 2359 (1983).

[35] H. P. Nilles, M. Srednicki and D. Wyler, Phys. Lett. B 124, 337 (1983);

[36] H. P. Nilles, M. Srednicki and D. Wyler, Phys. Lett. B 120, 346 (1983);

[37] A. Sen, Phys. Rev. D 30, 2608 (1984).

[38] S. Weinberg, Phys. Rev. Lett. 50, 387 (1983).

[39] R. L. Arnowitt, A. H. Chamseddine and P. Nath, Phys. Rev. Lett. 50, 232 (1983). 
[40] A. H. Chamseddine, P. Nath and R. L. Arnowitt, Phys. Lett. B 129, 445 (1983).

[41] P. Nath, R. L. Arnowitt and A. H. Chamseddine, "Model Independent Analysis Of LowEnergy Phenomena In Supergravity Unified Theories," NUB No: 2588, HUTP-83/A077 (unpublished 1983).

[42] D. A. Dicus, S. Nandi, X. Tata, Phys. Lett. B129, 451 (1983).

[43] D. A. Dicus, S. Nandi, W. W. Repko, X. Tata, Phys. Rev. Lett. 51, 1030 (1983).

[44] J. R. Ellis, D. V. Nanopoulos and K. Tamvakis, Phys. Lett. B 121, 123 (1983).

[45] L. Alvarez-Gaume, J. Polchinski, M. B. Wise, Nucl. Phys. B221, 495 (1983).

[46] L. E. Ibanez, C. Lopez, Phys. Lett. B126, 54 (1983).

[47] L. E. Ibanez and C. Lopez, Nucl. Phys. B 233, 511 (1984).

[48] L. E. Ibanez, C. Lopez and C. Munoz, Nucl. Phys. B 256, 218 (1985).

[49] R. L. Arnowitt, A. H. Chamseddine and P. Nath, "Supergravity and Unification," HUTP-83/A014, NUB 2597, Mar 1983. 34pp., Workshop on Problems in Unification and Supergravity, La Jolla, CA, Jan 1983. Published in La Jolla Unif. Wkshp.1983:0011 (QCD161:W61:1983)

[50] L. E. Ibanez, G. G. Ross, Phys. Lett. B110, 215-220 (1982).

[51] K. Inoue et al., Prog. Theor. Phys. 68, 927 (1982).

[52] R. Barbieri, S. Ferrara and C. A. Savoy, Phys. Lett. B119, 343 (1982).

[53] P. Fayet, Nucl. Phys. B 90, 104 (1975).

[54] S. Ferrara, D. V. Nanopoulos and C. A. Savoy, Phys. Lett. B 123, 214 (1983).

[55] L. E. Ibanez, Phys. Lett. B 118, 73 (1982).

[56] R. Barbieri, S. Ferrara, D. V. Nanopoulos and K. S. Stelle, Phys. Lett. B 113, 219 (1982).

[57] S. K. Soni and H. A. Weldon, Phys. Lett. B 126, 215 (1983).

[58] G. F. Giudice and A. Masiero, Phys. Lett. B 206, 480 (1988).

[59] R. L. Arnowitt and P. Nath, Phys. Rev. Lett. 69, 725 (1992).

[60] V. D. Barger, M. S. Berger and P. Ohmann, Phys. Rev. D 49, 4908 (1994).

[61] G. L. Kane, C. F. Kolda, L. Roszkowski and J. D. Wells, Phys. Rev. D 49, 6173 (1994).

[62] H. Baer and M. Brhlik, Phys. Rev. D 55, 3201 (1997) [hep-ph/9610224].

[63] P. Nath, R. L. Arnowitt, A. H. Chamseddine, "N=1 Supergravity Unified Theories And Their Experimental Signatures," published in "Supersymmetry and Supergravity, Nonperturbative QCD", Springer-Verlag, ed. P. Roy and V. Singh, 1984. p113-185.

[64] D. A. Kosower, L. M. Krauss, N. Sakai, Phys. Lett. 133B, 305(1983).

[65] T. C. Yuan, R. L. Arnowitt, A. H. Chamseddine and P. Nath, Z. Phys. C 26 (1984) 407. 
[66] A. Hoecker, Nucl. Phys. Proc. Suppl. 218, 189 (2011) [arXiv:1012.0055 [hep-ph]].

[67] K. Hagiwara, R. Liao, A. D. Martin, D. Nomura and T. Teubner, J. Phys. G G 38, 085003 (2011) [arXiv:1105.3149 [hep-ph]].

[68] S. Heinemeyer, D. Stockinger and G. Weiglein, Nucl. Phys. B 699, 103 (2004) [hep$\mathrm{ph} / 0405255]$.

[69] E. Eliasson, Northeastern University Preprint NUB No: 2621; Phys. Lett. B147, 65 (1984).

[70] J. R. Ellis, C. Kounnas and D. V. Nanopoulos, Nucl. Phys. B 247, 373 (1984).

[71] H. P. Nilles, Phys. Rept. 110, 1 (1984).

[72] H. E. Haber and G. L. Kane, Phys. Rept. 117, 75 (1985).

[73] S. Weinberg, Phys. Rev. D26, 287 (1982).

[74] N. Sakai and T. Yanagida, Nucl. Phys. B197, 533 (1982).

[75] S. Dimopoulos, S. Raby and F. Wilczek, Phys. Lett. B112, 133 (1982).

[76] J. Ellis, D. V. Nanopoulos and S. Rudaz, Nucl. Phys. B202, 43 (1982);

[77] R. L. Arnowitt, A. H. Chamseddine and P. Nath, Phys. Lett. B 156, 215 (1985).

[78] P. Nath, A. H. Chamseddine and R. L. Arnowitt, Phys. Rev. D 32, 2348 (1985).

[79] A. Bueno et al., JHEP 0704, 041 (2007) [arXiv:hep-ph/0701101].

[80] A. Rubbia, J. Phys. Conf. Ser. 171 (2009) 012020 [arXiv:0908.1286 [hep-ph]].

[81] H. Goldberg, Phys. Rev. Lett. 50, 1419 (1983).

[82] L. M. Krauss, Nucl. Phys. B227, 556 (1983).

[83] J. Ellis, J. S. Hagelin, D. V. Nanopoulos, K. Olive and M. Srednicki, Nucl. Phys. B238, 453 (1984).

[84] D. Feldman, Z. Liu and P. Nath, Phys. Rev. Lett. 99, 251802 (2007) [arXiv:0707.1873 [hep$\mathrm{ph}]]$.

[85] R. L. Arnowitt, B. Dutta, A. Gurrola, T. Kamon, A. Krislock, D. Toback, Phys. Rev. Lett. 100, 231802 (2008). [arXiv:0802.2968 [hep-ph]].

[86] E. Komatsu et al. [WMAP Collaboration], Astrophys. J. Suppl. 192, 18 (2011).

[87] E. Komatsu et al. [WMAP Collaboration], Astrophys. J. Suppl. 170, 377 (2007).

[88] E. Komatsu et al. [WMAP Collaboration], Astrophys. J. Suppl. 148, 175 (2003).

[89] F. R. Bouchet [ Planck Collaboration ], Mod. Phys. Lett. A22, 1857-1863 (2007).

[90] Z. Ahmed et al. [ CDMS Collaboration ], Phys. Rev. Lett. 102, 011301 (2009).

[91] Z. Ahmed et al. [ CDMS Collaboration ], Science 327, 1619 (2010).

[92] E. Aprile et al. [XENON100 Collaboration], arXiv:1104.2549 [astro-ph.CO].

[93] H. Baer and X. Tata, Phys. Lett. B 155, 278 (1985). 
[94] H. Baer, K. Hagiwara and X. Tata, Phys. Rev. Lett. 57, 294 (1986).

[95] P. Nath and R. Arnowitt, Mod. Phys. Lett. A 2 (1987) 331.

[96] H. Baer, C. -h. Chen, F. Paige and X. Tata, Phys. Rev. D 50, 4508 (1994) [hep-ph/9404212].

[97] S. Bornhauser, M. Drees, H. Dreiner, O. J. P. Eboli, J. S. Kim and O. Kittel, Eur. Phys. J. C 72, 1887 (2012) [arXiv:1110.6131 [hep-ph]].

[98] S. P. Martin, Perspectives on supersymmetry II, ed. G. L. Kane (World Scientific, Singapore, 1998), pp. 1-153, [hep-ph/9709356].

[99] H. Baer and X. Tata, Weak scale supersymmetry (Cambridge University Press, 2006).

[100] M. Drees, R. Godbole, and P. Roy, Sparticles (World Scientific, Singapore, 2004).

[101] G. G. Ross and R. G. Roberts, Nucl. Phys. B 377, 571 (1992).

[102] G. Herten, "ATLAS overview", Talk at SUSY2012-Beijing, University of Freiburg.

[103] G. Tonelli, "CMS overview", Talk at SUSY2012-Beijing, CERN.

[104] G. Aad et al. [ATLAS Collaboration], Phys. Lett. B 716, 1 (2012) [arXiv:1207.7214 [hep-ex]].

[105] S. Chatrchyan et al. [CMS Collaboration], Phys. Lett. B 716, 30 (2012) [arXiv:1207.7235 [hep-ex]].

[106] S. Akula, B. Altunkaynak, D. Feldman, P. Nath and G. Peim, Phys. Rev. D 85, 075001 (2012); [arXiv:1112.3645 [hep-ph]].

[107] J. Ellis and K. A. Olive, arXiv:1202.3262 [hep-ph].

[108] P. Candelas, G. T. Horowitz, A. Strominger and E. Witten, Nucl. Phys. B 258, 46 (1985). 